\title{
A concise biotyping system for differentiating strains of Escherichia coli
}

\author{
R GARGAN, W BRUMFITT, JMT HAMILTON-MILLER \\ From the Department of Medical Microbiology, Royal Free Hospital and Medical School, Pond Street, London NW3
}

SUMMARY A six test biotyping system comprising fermentation of dulcitol, sorbose, raffinose, and 5 ketogluconate, motility and production of $\boldsymbol{\beta}$-haemolysis was used to obtain biotype profiles for 514 strains of Escherichia coli isolated from the urinary tract. This profile was suffixed to the API-20E code, recorded when the strains were originally identified. An expanded and reliable biotyping system was thus created giving a greater number of possible biotypes than with either system alone. The sensitivity in terms of distinguishing the organisms studied was therefore greatly increased.

The use of O-serotyping in combination with biotyping is discussed. Biotyping can also be usefully supplemented by the determination of the eight commonest $E$ coli O-serotypes. This is of value in many clinical situations where differentiation of organisms is vital to the proper analysis of results.

Typing of organisms is fundamental to the understanding of problems associated with urinary tract infections. For example it is important to distinguish between failure to eradicate the organism (relapse) and the presence of a new infection by a different organism (reinfection). Additionally, it is important to establish the relation of the infecting organism to periurethral colonisation ${ }^{1}$ and to its presence in the faeces. ${ }^{2}$ The credibility of existing theories rests squarely upon the accuracy and reproducibility of the typing methods used. A complete serological analysis $(\mathrm{O}, \mathrm{H}$, and $\mathrm{K}$ antigens) of Escherichia coli would provide an accurate identification. This is impossible in practice, however, because of the time involved and the cost of a full range of antisera. Nevertheless, efforts to distinguish strains of $E$ coli have been made by using the eight commonest $\mathrm{O}$-serotypes, but this has proved unsatisfactory. Previous studies have shown ${ }^{3}$ that only about $63 \%$ of domiciliary strains and $45 \%$ of hospital strains would be identified by this means since in many cases the organisms are autoagglutinable or non-typable. Of the various typing systems available, study of the organisms' biological properties ("biotyping") appears a useful method of identification. It is technically simple and combined with $\mathrm{O}$-serotyping gives very precise identification if this is required. Thus biotyping and

Accepted for publication 30 June 1982 serotyping complement each other becausę experience shows that a particular O-serotype cak often be represented by a number of different biotypes. ${ }^{4}$

For a biotyping test to be maximally discriminatory $50 \%$ of strains must be positive and $50 \%$ negative and there are few simple tests that fulfil this basic criterion. However, for practical purposes, tests which are less than ideal have been shown to be suitable. Crichton and $\mathrm{Old}^{5}$ in a comprehensive study established a number of tests which were suitable and proved reliable for biotyping $E$ coli. A few of these tests are included in the API-20E system (API Laboratory Products Ltd) for the identification of Enterobacteriaceae. Since in this laboratory all coliforms are identified by the API-20E system our approach was to make it the basis for further biotyping. Thus to the API code were added the results of six further biotyping tests in order to expand the profile.

\section{Material and methods}

\section{PATIENTS AND ORGANISMS}

Five hundred and fourteen strains of $E$ coli were isolated from a total of 87 patients ( 85 women and 2 men) attending a urinary infection clinic over a period of three years. Two hundred and sixty strains were from mid-stream specimens of urine (MSU) from patients with a significant bacteriuria (defined as $\geqslant$ 
$10^{5}$ organisms/ml urine) and 240 isolates from the periurethral area of women who had been observed to be especially prone to recurrent infections. Thirteen organisms were isolated from the faeces of infected patients and one strain from prostatic fluid.

\section{BIOTYPING METHODS}

\section{API system}

All $E$ coli isolates were originally identified using the API-20E system following the manufacturer's instructions and colour chart precisely. The organisms were suspended in sterile distilled water and the incubation period was for $24 \mathrm{~h}$ at $37^{\circ} \mathrm{C}$.

\section{Fermentation tests}

Dulcitol, L-sorbose and D-raffinose (Southern Group Laboratories, Hither Green, London, UK) were dissolved at a concentration of $5 \mathrm{~g} / \mathrm{l}$ in $5 \mathrm{ml}$ amounts of peptone water with $2 \%$ Andrade's indicator and sterilised at $10 \mathrm{lb}$ psi for $10 \mathrm{~min}$. Potassium 5ketogluconate was prepared similarly but sterilised by filtration. One drop of a stock suspension (a single colony suspended in $5 \mathrm{ml}$ peptone water to give a population of approximately $10^{7}$ organisms $/ \mathrm{ml}$ ) was used to inoculate all tests. These fermentation tests were incubated at $37^{\circ} \mathrm{C}$ and read each day for three days. It should be stressed that any change in colour of the medium to red or pink was considered positive. As a result of experience gained from these daily readings it was decided that the best time for reading should be $48 \mathrm{~h}$ for dulcitol, sorbose and raffinose and $24 \mathrm{~h}$ for 5-ketogluconate.

\section{Production of beta-haemolysis}

A basal layer of $10 \mathrm{ml}$ Columbia Agar Base (Oxoid Ltd) was overlaid with $10 \mathrm{ml}$ of the same medium containing $7 \cdot 5 \%$ defibrinated horse blood. The stock suspensions were streaked out and incubated for $24 \mathrm{~h}$ at $37^{\circ} \mathrm{C}$. Any zone of beta-haemolysis around a well separated colony, viewed with transmitted light was considered positive.

\section{Motility}

The stock peptone water suspensions were incubated for $4-5 \mathrm{~h}$ at $37^{\circ} \mathrm{C}$ and motility investigated microscopically.

\section{Results}

For clarity, the results of coding strains with the API20 E system will be referred to as "API codes" and the results of fermentation tests, haemolysis and motility as "biotypes".

\section{API CODING}

Five hundred and fourteen isolates were identified as
Table 1 API codes and biotypes of 514 E coli strains. This can be seen to subdivide the API codes into biotypes

\begin{tabular}{lccl}
\hline API code & No of strains & \% total & $\begin{array}{l}\text { Different } \\
\text { biotypes }\end{array}$ \\
\hline 5144552 & 129 & 25 & 18 \\
5144572 & 108 & 21 & 19 \\
5044552 & 77 & 15 & 18 \\
5044572 & 20 & 4 & 8 \\
5144532 & 20 & 4 & 6 \\
5044542 & 18 & $3 \cdot 5$ & 6 \\
5144562 & 16 & 3 & 4 \\
4144532 & 16 & 3 & 3 \\
7144572 & 10 & 2 & 4 \\
(also 42 API codes & 100 & $19 \cdot 5$ & \\
with < 10 strains & & & \\
per code) & & & \\
\hline
\end{tabular}

$E$ coli using the API-20E system. Fifty-one different API codes were recorded. The majority of the strains (61\%) were grouped by three common API codes. The remaining 200 organisms were distributed among 48 different codes (Table 1). The tests with the greatest biotyping potential were: sucrose $-44 \%$ of strains positive, ornithine decarboxylase $(68 \%)$, melibiose $(88 \%)$, rhamnose $(90 \%)$ and lysine decarboxylase (93\%).

\section{BIOTYPING}

The majority $(72 \%)$ of the 514 strains fermented dulcitol; $66 \%$ sorbose; $46 \%$ raffinose and $49 \% 5$ ketogluconate. Most of the strains $(66 \%)$ were motile and $22 \%$ were beta-haemolytic (Table 2 ).

For ease of analysis we decided to code the six biotyping tests in two groups of three tests each with a single number for each group, following the system used for the API-20E. Thus, positive tests for dulcitol, sorbose and raffinose were coded 1,2 and 4 respectively; similarly, 5-ketogluconate, motility and beta-haemolysis. Eighty percent of the $E$ coli fell into 20 different biotypes (Table 3 ).

\section{REPRODUCIBILITY OF THE SCHEME}

Forty $E$ coli were isolated from the MSU of 20 patients with a significant bacteriuria on two consecutive occasions before treatment was started. These organisms were identified and coded by the

Table 2 Biotyping 514 strains of E coli, results of individual tests. (This summarises our biotyping scheme which supplements the API system)

\begin{tabular}{llll}
\hline Test & $\begin{array}{l}\text { Test read at } \\
\text { intervals }(h)\end{array}$ & $\begin{array}{l}\text { Strains } \\
\text { positive }\end{array}$ & \% positive \\
\hline Dulcitol & 48 & 370 & 72 \\
Sorbose & 48 & 337 & 66 \\
Raffinose & 48 & 234 & 46 \\
5 ketogluconate & 24 & 253 & 49 \\
Motility & $4-5$ & 338 & 66 \\
$\beta$-haemolysis & 24 & 111 & 22 \\
\hline
\end{tabular}


Table 3 Biotyping 514 strains of E coli, distribution of biotypes. This table shows the wide distribution of biotypes (The majority of API codes rarely exceeded 9 types (Table I) thus the possibility for discrimination is greatly increased)

\begin{tabular}{lll}
\hline Biotypes & No of strains & $\%$ total \\
\hline 20) biotypes with $\geqslant 10$ strains per biotype & 413 & 80 \\
2) biotypes with $<10$ strains per biotype & 101 & 20 \\
\hline
\end{tabular}

API scheme and then biotyped. The results were not compared until the survey had been completed. All paired API codes for the $40 \mathrm{E}$ coli tested were found to be the same. There was also good correlation for the biotypes. In only one pair was there disagreement in one test. It was also noted that changes in the API codes of consecutive $E$ coli isolates from the 87 patients (suggesting different biotypes) were generally paralleled by a change in the biotype pattern (confirming a different biotype). Thus, when we observed a total of 177 changes in the API codes of sequentially isolated $E$ coli from the individual patients, $162(92 \%)$ were accompanied by a change in the biotype.

\section{APPLICATION}

Escherichia coli strains with identical API codes and biotypes isolated from consecutive MSU from a number of patients indicated they were suffering recurrent relapses. For example, patient RM suffering from prostatitis had the strain (API code 5144552 , biotype 32 ) on five different occasions and all were serotyped as O6.

The biotyping system was also capable of differentiating repeated relapses from reinfection. Table 4 illustrates two different examples. Patient SD (a woman with a longstanding history of recurrent urinary infections) suffered two infections with different strains of $E$ coli in August and October 1978. Then in November an organism with the original biotype caused another infection which was followed by a relapse (with the same strain) two

Table 4 Results of biotyping and serotyping $E$ coli from infected patients

\begin{tabular}{lllll}
\hline Patient & \multicolumn{2}{l}{ Isolution date API code } & Biotype & Serotype \\
\hline SD & 30.8 .78 & 5144512 & 23 & O6 \\
& 30.10 .78 & 5144572 & 52 & NT $^{*}$ \\
& 14.11 .78 & 5144512 & 23 & O6 \\
SH & 28.11 .78 & 5144512 & 23 & O6 \\
& 5.4 .78 & 5044572 & 42 & NT \\
& 11.7 .78 & 5144552 & 37 & O6 \\
& 1.10 .79 & 5044552 & 11 & O11 \\
& 22.10 .79 & 5044572 & 61 & O6 \\
& 1.9 .80 & 5044552 & 02 & NT \\
& 2.10 .80 & 5044552 & 43 & O6 \\
\hline
\end{tabular}

*Non-typable. weeks later. Patient SH (a woman with a similar history) was repeatedly infected with different strains. Although a number of API codes corresponded, the biotypes indicated the organisms were all different strains. Here there was further confirmation by the demonstration of different $\mathrm{O}$-serotypes of several of the strains.

Results from this patient also illustrate that infections with organisms of the same serotype (three infections with O6) do not necessarily have the same API code or biotype and therefore are in fact different strains. Thus serotyping alone may give incomplete and erroneous information.

\section{Discussion}

The API-20E system was originally developed for identification to genus and species level. Only a few of the tests are useful for biotyping $E$ coli. However, even this limited biotyping ability has proved useful for "biotyping" strains of $E$ coli from $\mathrm{MSU}^{46}$ and also from other sources. ${ }^{7}$ Our three commonest API codes (Table 1) account for $61 \%$ of our $E$ coli strains. This figure is similar to that found by Davies ${ }^{4}$ $(50.5 \%)$. The rest of the strains fell into 48 other different API codes. This illustrates the usefulness of the API system by itself. Our API tests were read after $24 \mathrm{~h}$ (the upper limit of the time recommende of by the manufacturer). The 24 -hour incubation periodo appears to give more reproducible results than reading after overnight $(18 \mathrm{~h})$ incubation. ${ }^{8}$ Care was also taken to standardise the reading of the colour reactions and technical error was kept to a minimum by making sure that the team involved in the work always read all the tests. The reliability of the API system was confirmed when the codes of $E$ coli from 20 paired MSU were compared and were found to be identical. The biotyping profiles were also found to correlate well in the same paired MSUs. In agreement with Crichton and $\mathrm{Old}^{5}$ we obtained good typing with dulcitol, raffinose, sorbose and the motility test. In addition fermentation of 5 ketogluconate was found to give the best discrimination between strains. To our knowledge the use of this substrate for biotyping has not been reported before. Twenty-two percent of our strains were beta-haemolytic. Buckwold et al ${ }^{9}$ showed the production of beta-haemolysis to be a reproducible biotyping test and found $23 \%$ of their strains to be beta-haemolytic. As beta-haemolysis can be carried on a plasmid, ${ }^{10}$ changes in this characteristic should be viewed with caution. However, in none of our strains was the presence or absence of beta-haemolysis the only characteristic in which the biotype profile differed. We tested our strains for their avility to grow on minimal medium (prototrophy) and to hydrolyse aesculin but we did 
not consider these useful tests. Ninety-one percent of the strains grew on minimal medium and interpretation of aesculin hydrolysis was often equivocal.

Escherichia coli isolates having identical API codes were found to have a variety of biotype codes. Thus, greatly enchanced profiles were obtained when the biotypes - were added to the more commonly occurring API codes (Table 1). It was also reassuring that when we observed a change in the API code of sequential isolates from individual patients this was generally accompanied by a change in the biotype. This indicated that a change in the API code alone could be a reliable guide to differentiate strains.

Biotyping has proved useful as an adjunct to $\mathrm{O}$-serotyping in the particularly difficult situation where it is unclear whether patients having recurrent infections are suffering from reinfections (with different organisms) or relapses (with the same organism). When we compared the overall results of the O-serotypes of our strains with their biotypes, no relation was found. For example $E$ coli from various patients serotyped as $\mathrm{O} 6$ gave a wide range of different biotypes. However, where an individual patient had recurrent infections with $E$ coli of the same serotype they were often found to be the same biotype. This is understandable if the organisms were all of the same strain and this indicates the patient was suffering relapses. On the other hand, there were occasions where a different situation existed. For example, patient SH (Table 4) had infections with the same $\mathrm{O}$-serotype which were different biotypes. This means that the infections were with different strains and the patient was suffering reinfection.

The advantage of this biotyping system is that it is rapid, technically simple to assess, and is relatively inexpensive. Although the accuracy of the biotyping system is enhanced when used with API it is not essential as a partner in the combination. If desired the biotyping system which has been described could form a valuable adjunct for other typing schemes such as O-serotyping or resistotyping. Clearly by combining these studies an extremely reliable typing system can be guaranteed.

\section{References}

Stamey TA, Timothy M, Millar M, Mihara G. Recurrent urinary infections in adult women: the role of introital enterobacteria. Calif Med 1971;115:1-19.

${ }^{2}$ Grüneberg RN. Relationship of infecting urinary organism to the faecal flora in patients with symptomatic urinary infection. Lancet 1969;ii:766-8.

${ }^{3}$ Grüneberg RN, Leigh DA, Brumfitt WB. Escherichia coli serotypes in urinary tract infection: studies in domiciliary, antenatal and hospital practice. In: O'Grady F, Brumfitt W, eds. Urinary tract infection. London: Oxford University Press, 1968:68-79.

${ }^{4}$ Davies BI. Biochemical typing of urinary Escherichia coli strains by means of the API 20E Enterobacteriaceae system. J Med Microbiol 1977;10:293-8.

${ }^{5}$ Crichton PB, Old DC. Biotyping of Escherichia coli. J Med Microbiol 1979;12:473-86.

${ }^{\circ}$ Cicmanec JF, Evans AT. Classification of urinary tract infections by biotype identification of the pathogens. $J$ Urol 1980;124:689.

${ }^{7}$ Van der Waaij D, Tielemans-Speltie TM, de Roeck-Houben AMJ. Infection by and distribution of biotypes of enterobacteriaceae species in leukaemic patients treated under ward conditions and in units for protective isolation in seven hospitals in Europe. Infection 1977;5:188-94.

${ }^{8}$ Butler DA, Lobregat CM, Gavan TL. Reproducibility of the Analytab (API 20E) system. J Clin Microbiol 1975;2:322-5.

${ }^{9}$ Buckwold FJ, Ronald AR, Harding GKM, Marrie TJ, Fox L, Cates C. Biotyping of Escherichia coli by a simple multipleinoculation agar plate technique. J Clin Microbiol 1979;10:2758.

${ }^{10}$ Waalwijk C, Van Den Bosch JF, MacLaren DM, De Graaff J. A hemolysin plasmid, coding for virulence in a nephropathogenic Escherichia coli strain Antonie van Leeuwenhoek 1981;47:469.

Requests for reprints to: Professor W Brumfitt, Department of Medical Microbiology, Royal Free Hospital and Medical School, Pond Street, London NW3, England. 